\title{
Cut Detection in Remote Versatile Wireless Sensor Mobile Networks
}

\author{
K. Karthikeyan*, A. Leeladhar**, Ch. Elijah**, K. Manoj**, K. Laxman Babu** \\ * Associate professor, VIT University, Vellore, India \\ ** Students in School of Information Technology, India
}

\begin{tabular}{l} 
Article Info \\
Article history: \\
Received Jun 11, 2014 \\
Revised Nov 11, 2014 \\
Accepted Nov 23, 2014 \\
\hline Keyword: \\
Mobile Networks \\
Wireless Sensor \\
Remote Sensor Networks \\
(WSN)
\end{tabular}

\begin{abstract}
A remote sensor system can get differentiated into different joined segments because of the disappointment of some of its hubs, which is known as a "cut". In this project we consider the issue of catching cuts by the remaining hubs of a remote sensor system. We propose a calculation that permits (i) each hub to recognize when the integration to an exceptionally assigned hub has been lost, and (ii) one or more hubs (that are associated with the uncommon hub after the cut) to discover the event of the cut. The calculation is circulated and non concurrent: each hub needs to correspond with just those hubs that are inside its correspondence range. The calculation is focused around the iterative processing of an imaginary "electrical potential" of the hubs. The union rate of the hidden iterative plan is autonomous of the size and structure of the system. Remote Sensor Networks (Wsns) comprise of a huge number of small hubs having the ability of sensing, calculation, and remote correspondences. Remote sensor system can endure part issue in the system which is known as a cut. So a solitary topology of the system breaks into two or more parts. Here we examine a few slice recognition systems to discover the cuts in WSN.
\end{abstract}

Copyright @ 2014 Institute of Advanced Engineering and Science. All rights reserved.

Corresponding Author:

A. Leeladhar,

Students in School of Information Technology,

India.

Email: annepuleeladhar@gmail.com

\section{EXISTING SYSTEM}

Remote sensor system is made out of an influential base station and a set of low-end sensor hubs. Base station and sensor hubs have remote capacities and impart through a remote, multihop, specially appointed system. Remote sensor systems (WSN) have developed as an essential new innovation for instrumenting and observing the physical world. Remote sensor systems (Wsns) are an able situation for sensing extensive ranges at high spatial and positive determination. On the other hand, the minor size and minimal effort of the handling machines that makes them appealing for expansive arrangement additionally causes the loss of low operational dependability. Remote sensor systems (WSN) have risen as a vital new engineering for instrumenting and watching the physical world. The fundamental building square of these systems is a little microchip integrated with one or more MEMS (micro electromechanical framework) sensors, actuators, and a remote transceiver. A WSN is typically accumulation of hundreds or a huge number of sensor hubs. These sensor hubs are regularly thickly sent in a sensor field and can assemble information and course information once more to a base station (BS). A sensor has four essential parts: a sensing unit, a handling unit, a transceiver unit, and a force unit.

Wireless Multimedia Sensor Networks (WMSNs) has many challenges such as nature of wireless media and multimedia information transmission. Consequently traditional mechanisms for network layers are no longer acceptable or applicable for these networks. Wireless sensor network can get separated into multiple connected components due to the failure of some of its nodes, which is called a "cut". Existing cut 
detection system deployed only for wired networks.

Disadvantages:

1. Unsuitable for dynamic network reconfiguration.

2. Single path routing approach.

\section{PROPOSED SYSTEM}

Remote sensor systems (Wsns) are a guaranteeing innovation for observing vast areas at high spatial and transient determination Disappointment of a set of hubs will lessen the quantity of multi-bounce ways in the system. Such disappointments can result in a subset of hubs - that have not fizzled - to end up separated from the rest, bringing about a "cut". Two hubs are said to be disengaged if there is no way between them. We consider the issue of recognizing cuts by the hubs of a remote system. We accept that there is an uniquely assigned hub in the system, which we call the source node since a cut might possibly separate a hub from the source hub, we recognize two unique results of a cut for a specific hub. At the point when a hub $\mathrm{u}$ is detached from the source, we say that a DOS (Disconnected from Source) occasion has happened for $u$. When a cut occurs in the network that does not separate a node $\mathrm{u}$ from the source node, we say that CCOS (Connected, but a Cut Occurred Somewhere) event has occurred for $\mathrm{u}$. By cut detection we mean (i) detection by each node of a DOS event when it occurs, and (ii) detection of CCOS events by the nodes close to a cut, and the approximate location of the cut. In this article we propose a distributed algorithm to detect cuts, named the Distributed Cut Detection (DCD) algorithm. The algorithm allows each node to detect DOS events and a subset of nodes to detect CCOS events. The algorithm we propose is distributed and asynchronous: it involves only local communication between neighboring nodes, and is robust to temporary communication failure between node pairs The convergence rate of the computation is independent of the size and structure of the network.

\section{MODULE DESCRIPTION}

\subsection{Distributed Cut Detection}

The algorithm allows each node to detect DOS events and a subset of nodes to detect CCOS events. The algorithm we propose is distributed and asynchronous: it involves only local communication between neighboring nodes, and is robust to temporary communication failure between node pairs. A key component of the DCD algorithm is a distributed iterative computational step through which the nodes compute their (fictitious) electrical potentials. The convergence rate of the computation is independent of the size and structure of the network.

\subsection{Cuts in Wireless Sensor Networks}

ONE of the novel difficulties in portable adhoc organizing situations is the wonder of system parceling, which is the breakdown of an associated system topology into two or more separate, separated topologies [3]. Similarly sensors get to be fizzle for a few reasons and the system may breaks into two or more partitioned segments so can say that when various sensor comes up short so the topology changes. A hub may fizzle because of a mixed bag of conditions, for example, mechanical or electrical issues, ecological debasement, and battery decrease. Truth be told, hub disappointment is relied upon to be truly regular abnormality because of the normally constrained vitality stockpiling of the hubs that are controlled by little batteries. Disappointment of a set of hubs will decrease the quantity of multihop ways in the system. Such disappointments can result in a subset of nodes that have not failed to become disconnected from the rest of the network, resulting in a partition of the network also called a "cut". Two nodes are said to be disconnected if there is no path between them. And As we know that sensors has Disconnectivity from the network is normally referred as a partition of the network of cut in the wireless sensor network, which arise many problems like unreliability ,data loss, performance degradation. Because of cuts in wireless sensor network many problems may arise like a wired network means data loss problem arises, means data reach in a disconnected route.

\subsection{Problems Due to Cuts}

As mentioned above if any node breaks down then the network is separated into different parts so the topology of the network changes but still network works. But because partition affects reliability, data loss, QOS of the network, efficiency, data processing speed. Because if any data passes unfortunately in a wrong route so data loss occurs this also shows unreliability of the network. 


\section{SOURCE NODE}

We consider the problem of detecting cuts by the nodes of a wireless network. We assume that there is a specially designated node in the network, which we call the source node. The source node may be a base station that serves as an interface between the network and its users. Since a cut may or may not separate a node from the source node, we distinguish between two distinct outcomes of a cut for a particular node.

\subsection{Cuts in Sensor Networks}

Consider a set $\mathrm{S}$ of $\mathrm{n}$ sensors, which are modeled as points in the two-dimensional plane. (More generally, we can assume that the sensors lie on a surface or terrain that is topologically equivalent to the plane). An adversary can make a linear cut through the sensor network, disabling all the sensors on one side of the line; the base station is assumed to lie on the other (safe) side. Formally, given a line L, let L- and L+ denote the two half-planes defined by $\mathrm{L}$, and let $\mathrm{L}-(\mathrm{S})$ and $\mathrm{L}+(\mathrm{S})$ denote the subset of sensors that lie in these half-planes. We will adopt the convention that the linear cut induced by L disables all the sensors in L-(S).

Alternatively, the adversary can disrupt the communication so that sensors on one side of the line cannot communicate with sensors on the other side, including the base station. These two formulations are equivalent for our purpose. There are other natural forms of cuts, such as circular cuts, rectangular cuts, polygonal cuts.

We a We call a direct cut a "-cut if at any rate " part of the sensors are cut off, where $0<€<1$ is a client determined parameter. Our essential concentrate in this paper is to create a low-overhead plan for identifying $€$-cuts in sensor systems.

Our plan for identifying $€$-cuts will pick a little subset of sensors, which go about as sentinels. Every sentinel will correspond with the base station at a standard time interim. We expect that the base station is not assaulted, and it generally lies in the safe half plane L+. A correspondence disappointment from a sentinel is taken to imply that the sentinel has been cut off. Our issue now gets to be: can one pick a little number of sensor hubs as sentinels so that (1) each $€$-cut can be distinguished built exclusively in light of the live/dead status of sentinels, and (2) the calculation does not report false positives. Assume we demonstrate an accumulation of 1000 sensor hubs, dispersed consistently at irregular, and its sentinel set for $"=0.05$. Before portraying our results, we first quickly examine why we picked $€$-cuts as our definition, why keeping away from false positives is testing, and why the identification plan requires an estimate slack.

\section{2. $€$-Cuts}

The $€$-cuts are persuaded both by down to earth and hypothetical concerns. It bodes well for treat disappointments as critical just when a small amount of the system is cut. It might be enticing to request plans that discover disappointment of a settled (client determined) number of sensors, paying little heed to the system size. In any case, no effective and versatile arrangement is hypothetically conceivable for this situation, as the accompanying straightforward illustration shows. Envision n sensors orchestrated around, and assume we need to locate cuts of size $\mathrm{m}$. At that point, no less than one sensor for each $\mathrm{m}$ sequential sensors must be picked as a sentinel, which scales crudely with the system size.

\subsection{False Positives}

By checking sufficiently a lot of people arbitrarily picked sensors, one can identify all $€$-cuts with high likelihood. For example, an arbitrary specimen of size $O(1 / € \log 1 / \delta €)$ is sufficient to get any "-cut with likelihood no less than $1-\delta[21]$, [22]. The calculation basically proclaims a $€$-cut at whatever point no less than one of the picked sensors falls flat. Lamentably, this basic plan experiences the false positives issue. Numerous cuts reported by this calculation, nonetheless, are false positives, where the measure of the fizzled system can be self-assertively littler than $€ n$. To be sure, if one of the irregular examples happens to lie on the limit of the sensor field, then it can result in a caution regardless of the fact that a solitary sensor is cut off. A more advanced manifestation of inspecting can adequately dispense with false positives, yet at the cost of an expansive number of sentinels. Specifically, the idea of $€$-rough guess can be utilized to recognize all cuts bigger than $€ n$ and those littler than, say, $1 / 2 € n$. However a $€$-close estimation requires $£(1 / € 2 \log 1 \delta)$ sentinel hubs. A straightforward computation, including the real constants included, nonetheless, demonstrates that actually for unobtrusive estimations of $€=0.1$ and $\delta=0.05$, the span of the sentinel set is no less than 10,000! Hence, arbitrary inspecting based plans are infeasible, because of false positives or because of unsalable substantial size.

Lemma: In a game plan of $n$ lines in the plane, there is dependably a level of size at most $6 \mathrm{n}$ between the levels $5 / 6 € n$ and $€ n$. Essentially, there is dependably a level of size at most $4 \mathrm{n}$ between levels Confirmation: The aggregate many-sided quality of the first $€ n$ levels is at generally $€ n 2$. Plainly, this is likewise an upper bound on the aggregate many-sided quality of the $1 / 6 € n+1$ levels between levels $€ n$ and $5 / 6 € n$. By the pigeon gap guideline, no less than one of these levels must have size at most $€ n 2 /(1 / 6 € n+1) 6 n$. 
A comparable to contention demonstrates that there is a level of size at most $4 \mathrm{n}$ between levels $2 / 3 € \mathrm{n}$ and $1 / 2 € n$. We can now finish the evidence of Theorem.

Evidence: Consider an plan of $\mathrm{n}$ lines in the plane.

Pick an and $b$ such that $1 / 2 € n<a<2 / 3 € n<b<€ n$, and the extent of the a level is at most $4 n$ and the measure of the b-level is at most $6 \mathrm{n}$; such an and $\mathrm{b}$ exist by the former lemma. The aggregate size of these two levels is at most $10 \mathrm{n}$, and $(\mathrm{b}-\mathrm{a}+1), 1 / 6 € \mathrm{n}$. By Lemma, we infer that there is a crisscross way of size $\mathrm{O}(1 €)$ between levels an and $\mathrm{b}$. This crisscross way is unmistakably a separator between the $€ n$ and the $1 / 2 € n$ levels.

The steady elements in Theorem are detached. Our essential objective is just of demonstrate the asymptotic come about that sentinel sets of size $\mathrm{O}(1 €)$ exist.

\subsection{Detecting $€$-cuts from a Signature}

The $€ n$ sensors that are cut off may lie either below or above the line. We, therefore, compute two separators, one to detect separation of points below the cutting line, and the other to detect separation above the line. In order to avoid unnecessary replication, we describe our scheme for the lower separator, with the understanding that the complete construction involves a symmetric application of the algorithm for the other case as well.

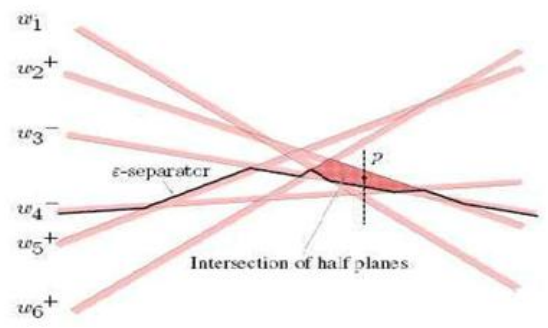

Figure 1. The Intersection of the half-planes determined by the sentinel lines is a cell of the arrangement

\section{NETWORK SEPERATION}

Failure of a set of nodes will reduce the number of multi-hop paths in the network. Such failures can cause a subset of nodes - that have not failed - to become disconnected from the rest, resulting in a "cut". Because of cut, some nodes may separated from the network, that results the separated nodes can't receive the data from the source node.

\section{CONCLUSION}

In this article we discuss WSN cuts and existing cut detection schemes in WSN. Wireless Sensor Networks (WSNs) often suffer from disrupted connectivity caused by its numerous aspects such as limited battery power of a node and unattended operation vulnerable to violent interfering. And this loosing connectivity is often referred as a network cut sometimes. In this paper, we studied several schemes of detecting cuts and we conclude by stating that cuts in WSN are a big problem which may introduce some unreliability in the network. So it is necessary to identify and detect cuts in WSN. To the best of our knowledge and based on our studies and reviews, no useful and efficient cut detection scheme has been proposed and implemented so far.

\section{REFERENCES}

[1] G. Dini, et al., "An algorithm for reconnecting wireless sensor network partitions," In European Conferenceon Wireless Sensor Networks, 2008, pp. 253-267.

[2] N. Shrivastava, et al., "Detecting cuts in sensor networks," ACM Trans. Sen. Newt., 2008, Vol. 4, No. 2, pp. 1-25.

[3] H. Ritter, et al., "A partition detection system for mobile ad-hoc networks," In First Annual IEEE Communications Society Conference on Sensor and Ad Hoc Communications and Networks (IEEE SECON 2004), 2004, pp. 489-497.

[4] M. Hauspie, et al., "Partition detection in mobile ad-hoc networks," in 2nd Mediterranean Workshop on Ad-Hoc Networks, 2003, pp. 25-27.

[5] P. Barooah, "Distributed cut detection in sensor networks," In 47thIEEE Conference on Decision and Control, 2008, pp. $1097-1102$.

[6] A. D. Wood, et al., "Jam: A jammed-area mapping service for sensor networks," In IEEE Real Time System Symposium, 2003. 